\title{
Hippocampal Serotonin Mediates Hypoactivity in Dietarily Obese Hamsters: A Possible Manifestation of Aging?
}

\author{
KATARINA T. BORER, ${ }^{1}$ RANDY BONNA AND MICHAEL KIELB
}

Department of Kinesiology, The University of Michigan, Ann Arbor, MI 48109

\author{
Received 21 March 1988
}

\begin{abstract}
BORER, K. T., R. BONNA AND M. KIELB. Hippocampal serotonin mediates hypoactivity in dietarily obese hamsters: A possible manifestation of aging? PHARMACOL BIOCHEM BEHAV 31(4) 885-892, 1988.-To determine whether endogenous opiates mediate hyperactivity in food restricted hamsters and serotonergic fibers innervating the hippocampus mediate hypoactivity in obese hamsters, food restriction and high-fat diet supplementation were used to produce significant body fat changes ( $8 \mathrm{vs.} 21 \%$ ). The levels and pattern of spontaneous running were examined after IP saline or naloxone $\mathrm{HCl}$ $(20 \mathrm{mg} / \mathrm{kg})$ and following the infusion of vehicle and 5,7-dihydroxytryptamine creatine sulfate $(4 \mu \mathrm{g} / 2 \mu \mathrm{l})$ into rostromedial septum of mature female hamsters. Septum-medial preoptic area (POA), hippocampus, hypothalamus, and cortex were dissected from the three groups as well as from two additional groups of hamsters receiving vehicle or neurotoxin. Concentrations of serotonin, norepinephrine, and dopamine were measured in these tissues by HPLC method. Fat-fed hamsters were hypoactive relative to food-restricted hamsters. Naloxone had no significant effect on running behavior. Serotonin neurotoxin increased the running activity of fat-fed hamsters to the level displayed by control hamsters by increasing the number of runs, the total activity level, the speed of running and by decreasing the duration of pause:s. Neurotoxin led to selective deletion of serotonin in the hippocampus (77\%) and parietal cortex (50\%). Serotonergic fibers innervating the hippocampus thus appear to mediate the hypoactivity that is induced by dietary obesity in mature hamsters. Since serotonin mediates some other manifestations of aging, and slow weight increases characterize mid-portion of hamster life span, we hypothesize that serotonergic mediation of hypoactivity is another manifestation of aging.
\end{abstract}

Fasting Hyperactivity Naloxone Limbic forebrain

MECHANISMS which regulate body energy balance and control spontaneous running behavior are interrelated. This is apparent at the time of sexual maturation in rodents when there is a rapid increase in spontaneous running activity to an all-time high at the same time as the rate of somatic growth and of weight gain rapidly decline (8). It is also evident after the onset of sexual maturity, during the period of slow weight gain when activity levels decline in inverse proportion to increases in body size $(4,8,37)$. Thus, when the weight of mature hamsters doubles, their spontaneous activity levels are reduced by about $50 \%(4,8)$. Furthermore, when accumulation of excess energy results in obesity, mice $(45)$, rats $(37,40)$, and hamsters (5) display even greater spontaneous hypoactivity.

Reciprocal relationship between energy regulation and spontaneous running remains intact after some neurosurgical manipulations which affect both mechanisms. In the hamster, voluntary running levels are reduced by $80 \%$ following electrocoagulative lesions of rostromedial septum (10), hori- zontal transection of septo-hippocampal interconnections (9), or bilateral transections of dorsal hippocampus (11), while at the same time these neurosurgical procedures induce striking acceleration of somatic growth and some accumulation of body fat. These observations suggest that septo-hippocampal neural circuits participate in the bioenergetic modulation of spontaneous running in hamsters in that they suppress somatic growth and accumulation of fat at the same time that they stimulate spontaneous running activity.

That this reciprocal relationship is not merely coincidental and consequent to physical proximity of two neural circuits mediating age-associated changes in the regulation of energy balance and spontaneous running is shown by restricting the access to food energy in rodents. When a negative energy balance is imposed on rats $(17-19,32)$, hamsters $(5,6)$, gerbils $(17)$, and kangaroo rats $(23)$, their spontaneous running rises in inverse proportion to decreases in body weight until debilitation or death occur. of Michigan, Ann Arbor, MI 48109. 
All of the above evidence suggests that spontaneous running is functionally linked to mechanisms regulating energy balance in a nonhomeostatic fashion. When the energy content of the body declines below the norm, the resultant hyperactivity exacerbates the negative energy balance. On the other hand, when the energy content of the body is driven above the norm, the concomitant hypoactivity enhances and perpetuates obesity. This nonhomeostatic reciprocal relationship between body energy content and spontaneous activity has also been observed in humans where spontaneous hypoactivity has been reported in obese individuals $(2,16)$, and hyperactivity has been reported to accompany compulsive dieting and excessive weight loss (3, $26,30,44)$.

Our intent in this study was to clarify the neurochemical basis of the brain mechanism which interrelates energy homeostasis with spontaneous running. We have relied on previous findings linking endogenous opiates with facilitation of spontaneous running in female hamsters (34) and linking septo-hippocampal serotonergic neurons with hypoactivity in female rats $(42,43)$ to form our hypotheses. Our experiments were designed to test two hypotheses, first that endogenous opiates are necessary for expression of hyperactivity in hamsters which are in negative energy balance, and second, that septo-hippocampal serotonergic neurons induce hypoactivity in hamsters which have become obese. We used food restriction and dietary high-fat supplements to produce deviations in hamster body energy balance from its norm, and opiate receptor blockade and nuerochemical destruction of septal serotonergic neurons to assess the relative contribution of endogenous opiates and septohippocampal serotonin neurotransmission in nutritional modulation of spontaneous running.

METHOD

\section{Animals}

Thirty-three female golden hamsters (Mesocricetus auratus, Harlan Sprague-Dawley, Haslett, MI), between 174 and 241 days old, were used.

\section{Maintenance}

Animals were housed individually in metal cages with wire floors ( $20 \mathrm{~cm}$ high, $20 \mathrm{~cm}$ wide, and $28 \mathrm{~cm}$ high) at all times except when they were tested for spontaneous running levels. Animal room temperature was maintained at $20-22^{\circ} \mathrm{C}$ and lighting was 14 L: $10 \mathrm{D}$, lights on at $7 \mathrm{a} . \mathrm{m}$.

\section{Experimental Design}

In Experiment 1, nineteen hamsters were assigned to one of three dietary groups for a period of 66 days: control group $(n=6,136.7 \pm 2.7 \mathrm{~g})$, food-restricted group $(n=6,135.9 \pm 2.0$ $\mathrm{g})$, or fat-diet supplemented group $(\mathrm{n}=7,139.3 \pm 1.5 \mathrm{~g})$. Adaptation to running cages and measurements of running activity following intraperitoneal saline or naloxone injections took place between days 16 and 46 of the experiment. Saline and naloxone injections were administered in randomized order and were separated by one day of no injection.

Between days 48 and 50 , the vehicle $(0.1 \%$ ascorbate) was injected into the rostromedial septum to determine the possible effect of nonspecific intraseptal fluid administration on voluntary running. The effects of infusion were tested on the third day postinjection.

Between days 58 and 60 , serotonin neurotoxin 5,7- dihydroxytryptamine creatine sulfate was injected into the rostromedial septum of all hamsters. Voluntary activity levels and running patterns were examined on the third day postinjection when similar neurotoxic lesions were reported to produce maximal behavioral consequences in rats (42).

On day 67 of the experiment, hamsters were killed by decapitation between $9: 30$ and $12 \mathrm{a} . \mathrm{m}$. Their brains were dissected for regional determination of serotonin, norepinephrine, and dopamine concentrations as a function of nutritional variables. Carcasses were frozen for subsequent body fat determination.

Since in Experiment 1 hamsters served as their own controls and all received serotonin neurotoxin following the administration of vehicle, Experiment 2 was performed for comparison of regional concentrations of brain serotonin in hamsters which received serotonin neurotoxin $(n=7$, $167.0 \pm 8.9 \mathrm{~g}$ ) and others which received intraseptally the vehicle $(n=7,165.0 \pm 6.9 \mathrm{~g})$. On the third postinfusion day, animals were killed by decapitation, and their brains were dissected for regional determination of serotonin, norepinephrine, and dopamine concentrations.

\section{Running Activity}

For measurements of running activity hamsters were housed in acrylic boxes $(38 \mathrm{~cm}$ high, $38 \mathrm{~cm}$ wide, and $49 \mathrm{~cm}$ long) with wire floors and a horizontal disc exerciser, $25-\mathrm{cm}$ in diameter. Two days of familiarization with the running box preceded the first drug treatment and data collection. For all subsequent tests hamsters were housed in the wheel box during a 24-hour period immediately following intraperitoneal saline or naloxone injections or 48 hours following intraseptal vehicle or neurotoxin infusions.

Disc turns were recorded on electromechanical counters and on Harvard cumulative event recorders. Total number of wheel turns, number and duration of running bouts, running speed, and duration of pauses were determined from the event records. Paper speed on the event recorder was 5 $\mathrm{mm} / \mathrm{min}$, and each disc turn produced a $0.25 \mathrm{~mm}$ sideway pen excursion. To separate the infrequent accidental activations of the wheel from actual running, running bouts were defined as episodes of running activity consisting of more than 20 consecutive disc revolutions separated by pauses, that is periods of more than 150 seconds of inactivity. Running speed was determined from the rate of sideways pen excursion during an individual running bout. Total nocturnal running volume was a sum of activity during all of the running bouts.

\section{Nutritional Manipulations}

All hamsters received water ad lib. Control group received Purina Formulab 5008 powder ad lib inside 4-oz glass jars during the first 24 days and the same diet in the form of pellets thereafter. Food-restricted group received the powdered diet in quantities which were adjusted to $70 \%$ of the ad lib intake at the start of the nutritional manipulation and which were increased to $85 \%$ of ad lib intake until the weight of the animals stabilized at $80 \%$ of control weights. Fat-fed group received a choice of pellets and of a high-fat diet consisting of a mixture of $60 \%$ vegetable shortening and $40 \%$ of chow powder inside a 4-oz glass jar.

According to manufacturer, Purina Formulab 5008 chow contains $23 \%$ protein, $6.5 \%$ fat, and $49.9 \%$ carbohydrate, and $4.3 \mathrm{kcal} / \mathrm{g}$ in gross energy content. We determined the gross energy content of chow to be $4.1 \mathrm{kcal} / \mathrm{g}$ by bomb 
calorimetry. Solid vegetable shortening contained $9.2 \mathrm{kcal} / \mathrm{g}$ of gross energy. Food intakes were measured during the first 27 days of the experiment to assess their contribution to changes in body mass.

\section{Body Weight and Fat Measurements}

Hamsters were weighed daily to monitor the effectiveness of nutritional manipulations. Body fat was determined (27) from carcasses after removing the brain for neurochemical analysis and after discarding the gut content. Carcasses were autoclaved, homogenized in $250 \mathrm{ml}$ of water, and dehydrated by freeze-drying. Dehydrated matter was ground to powder. The fat content of an aliquot of powdered dehydrated homogenate was extracted with petroleum ether.

\section{Drug Administration}

Naloxone $\mathrm{HCl}$ (E. I. Du Pont de Nemours, Wilmington, DE) was injected IP at the dose of $20 \mathrm{mg} / \mathrm{kg}$ previously found to inhibit voluntary running but not other incentive behaviors (12). An equivalent volume of saline was injected IP either $48 \mathrm{hr}$ before or $48 \mathrm{hr}$ after naloxone injection. Injections were done during the hour before the onset of nocturnal running.

The vehicle and the neurotoxin were infused from a $10 \mu \mathrm{l}$ Hamilton syringe through a length of PE-10 polyethylene tubing and through a 30-ga stainless-steel cannula lowered stereotaxically into the rostromedial septum under sodium pentobarbital anesthesia $(75 \mathrm{mg} / \mathrm{kg})$. With the skull level between the bregma and lambda, a midline hole was trephined in the skull $2 \mathrm{~mm}$ anterior to bregma, and the cannula tip was lowered down the midline, $2 \mathrm{~mm}$ anterior to bregma and 5 $\mathrm{mm}$ below the dura. These coordinates define the rostromedial septum-medial preoptic area at the level of anterior commissure where electrocoagulative lesions induce hypoactivity, and accelerate growth (10).

5,7-Dihydroxytryptamine creatine sulfate (DO136, Sigma) was dissolved in $0.1 \%$ sodium ascorbate and $4 \mu \mathrm{g}$ of free base in $2 \mu \mathrm{l}$ was infused into the rostromedial septum. To prevent the nonspecific norepinephrine depletion, norepinephrine reuptake was blocked by injection of desipramine $\mathrm{HCl}(25 \mathrm{mg} / \mathrm{kg}$, IP) $67.5 \pm 4.6 \mathrm{~min}$ before the vehicle and $54.0 \pm 2.3 \mathrm{~min}$ before the neurotoxin infusion. The intraseptal infusion rates were $0.37 \pm 0.03 \mu \mathrm{l} / \mathrm{min}$ for the vehicle and 0.26 $\mu \mathrm{l} / \mathrm{min}$ for the neurotoxin. After the infusion, cannula was left in place 2 min before retraction to prevent fluid migration out of the injection site.

\section{Tissue Dissection}

Brain was dissected from the skull and dura and immersed in ice-cold saline within 40.6 \pm 2.1 seconds of decapitation in Experiment 1 and within $37.4 \pm 2.0$ seconds in Experiment 2 . Brain was dissected on an ice-chilled aluminum bloc by a modification of the procedure of Brownstein et al $(13,14)$. A 3-mm wide strip of parietal cortex was separated on both sides of the brain from the corpus callosum and the cerebral hemispheres by cuts parallel to longitudinal fissure. The hippocampus on both sides of the brain was severed at its transition into fornix. The brain was then placed with the ventral surface up and divided by three coronal cuts, through the anterior third of olfactory tubercle, optic chiasm, and the posterior border of the median eminence. Septum-medial preoptic area (POA) block was defined by the first two coronal cuts, and hypothalamus by the second and third coronal cuts. Lateral borders of the two blocks of tissues were parasagittal cuts passing through lateral ventricles, and dorsal borders were cuts placed just dorsal to the anterior commissure.

Tissues were rapidly weighed on a Cahn torsion electrobalance, placed into plastic vials and snap-frozen in isopentane and dry ice within $9.3 \pm 0.2 \mathrm{~min}$ of decapitation in Experiment 1 and within 6.4 $0.4 \mathrm{~min}$ in Experiment 2. Cortical and hippocampal tissues collected from both sides of brain and single septal and hippocampal blocks of tissue were stored at $-70^{\circ} \mathrm{C}$ for subsequent neurochemical analyses. Neurochemical analyses were performed after 8.5 months of storage for tissues from Experiment 1 and after 1 month of storage for tissues from Experiment 2.

\section{Assay Methods}

A coulometric detector (ESA Coulochem model 5100, Medford, MA) with two analytical cells (ESA model 5011) was used for determination of regional concentrations of brain monoamines by reverse-phase high-performance liquid chromatography. Detectors were set at 0 and $0.4 \mathrm{~V}$, respectively.

Tissues were disrupted by sonication (Heat Systems Ultrasonics sonicator model W185F, setting 2.5) for $10-15 \mathrm{sec}$ in homogenization buffer $(0.16 \mathrm{~N}$ perchloric acid, $0.4 \mathrm{mM}$ sodium bicarbonate) and with DHBA (3,4-dihydroxybenzilamine $\mathrm{HCl}$, Sigma D7012, $\left.4.6 \times 10^{-7} \mathrm{M}\right)$ and methylserotonin (N-omega-methyl-5-hydroxytryptamine oxalate, $3.6 \times 10^{-7}$ M) as internal standards. Homogenates were centrifuged 15 min at $1^{\circ} \mathrm{C}$ at $12,300 \times \mathrm{g}$ in a model $\mathrm{J} 21 \mathrm{C}$ Beckman centrifuge and the supernatant was injected onto an Altec C-18 ultrasphere column $(4.6 \mathrm{~mm}$ i.d., $25 \mathrm{~cm}$ long) without further treatment. The mobile phase was a modification of a Rainin protocol (35) and consisted of $0.09 \mathrm{M}$ monochloroacetic acid, $0.56 \mathrm{mM}$ EDTA, $0.35 \mathrm{mM}$ sodium octyl sulfonate and 18 to $22.5 \%$ methanol at $\mathrm{pH} 3.5$.

An integrator (Spectraphysics, model SP4270) was used for peak height (norepinephrine, NE) and area (serotonin, 5HT and dopamine, DA) detection. The concentrations of indoleamines were calculated by comparing sample peak area or height with those of standard solutions. All peak areas or heights were normalized to the internal standards (DHBA for the catecholamines, M5-HT for 5-HT) and no corrections were made for recovery.

\section{Statistical Analysis of Data}

Analysis of variance (ANOVA) with repeated measures was used in evaluation of body weight changes with time. ANOVA and analysis of covariance with repeated measures was used to assess the effects of nutritional and pharmacological manipulations on the level and pattern of spontaneous running. In addition, paired $t$-test was used to compare the level and pattern of running before and after drug administration. Disc turns were converted to distance using $48 \mathrm{~cm}$ as representative of average circumference of a hamster running path on a disc (4). When determinations of neurotransmitter concentrations were done bilaterally, mean values were used in statistical analyses. ANOVA and Duncan's multiple range test (24) were used in the evaluation of the nutritional and neurotoxic treatments on brain neurochemistry in Experiment 1. Student's $t$-test for independent groups was used to compare ad lib food intakes of control and fat-fed hamsters and for neurochemical comparisons in Experiment 2. 


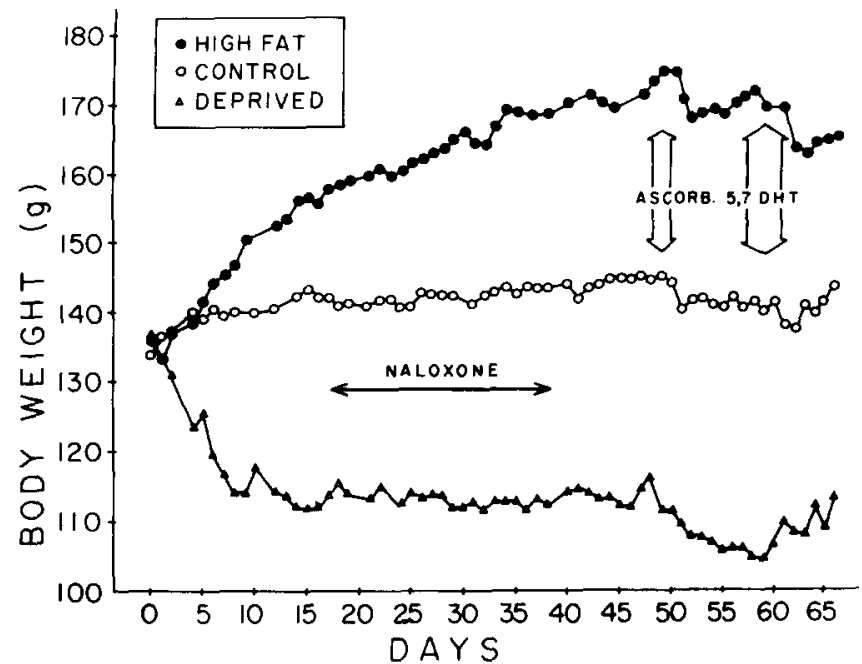

FIG. 1. Changes in body weight as a function of 66-day exposure to chow diet (control hamsters, $n=6$ ), to restricted access to chow (deprived hamsters, $n=5$ ), and to choice between chow and high-fat $\operatorname{diet}(60 \%$ shortening, $40 \%$ chow, $n=7)$.

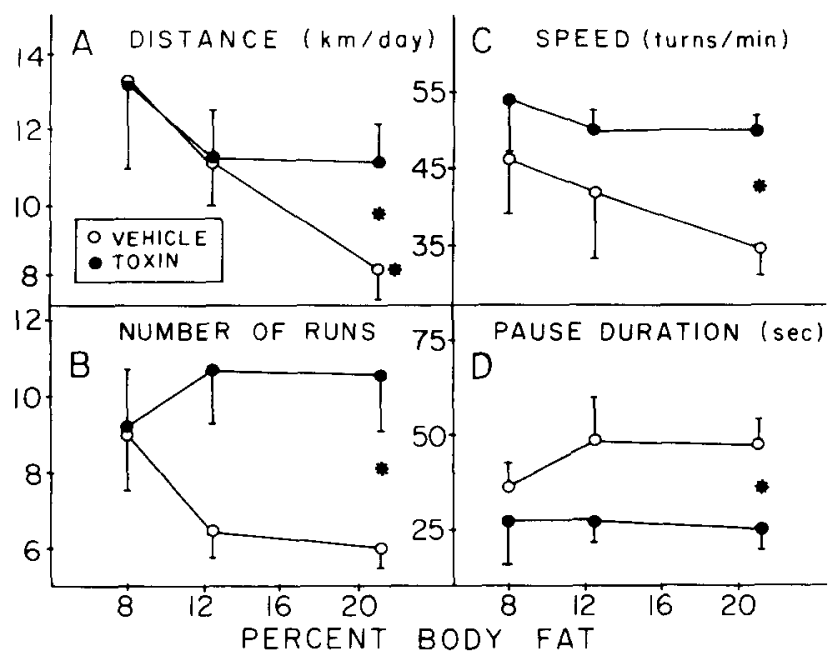

FIG. 2. Effect of intraseptal administration of 5,7-dihydroxytryptamine creatine sulfate in food-restricted hamsters ( $8 \%$ body fat), control diet hamsters ( $12.5 \%$ body fat) and fat-fed hamsters (21.3\% body fat) on total running activity levels (A), number of running bouts (B), speed of running $(C)$, and duration of pauses between the running bouts (D). Asterisks between the curves denote significant differences as a function of neurotoxin treatment, and besides the values, significant differences as a function of nutritional manipulations. Brackets denote S.E.M.s.

\section{RESULTS}

\section{Nutritional Variables}

Between days 2 and 9, fat-fed hamsters consumed $30 \%$ more calories $(57.4 \pm 4.3)$ than the control hamsters $(44.1 \pm 4.7 \mathrm{kcal} / \mathrm{day}, p<0.05)$. Intakes of the two groups did not differ during the remainder of the 27-day measurement period.

Figure 1 illustrates dietary influence on body weight. Following a 2-week period of weight loss, the weight of foodrestricted hamsters stabilized at about $80 \%$ of the control hamsters' weight. Body weight of restricted hamsters was first significantly lower $(123.3 \pm 2.8 \mathrm{~g}, p<0.01)$ than that of control hamsters $(140.4 \pm 2.6)$ on day 4 of deprivation.

After the initial 2-week period of rapid weight gain, fat-fed hamsters continued to gain weight at a somewhat faster rate than the control hamsters for the next five weeks before returning to control rates of weight gain. The weight of fatfed hamsters $(151.3 \pm 3.8, p<0.05)$ first exceeded that of control hamsters on day 10 of dietary manipulation.

Final body fat content of fat-fed hamsters $(21.3 \pm 2.3 \%$, $p<0.01$ ) was significantly higher than that of the other two groups. However, body fat contents of control hamsters $(12.5 \pm 1.6 \%)$ and food restricted hamsters $(8.0 \pm 1.7 \%)$ were not significantly different.

\section{Nutritional Modulation of Spontaneous Running}

The total amount of running activity increased by about $20 \%$ in food-restricted hamsters, and decreased by about $24 \%$ in fat-fed hamsters. Activity levels of hyperactive foodrestricted hamsters differed significantly from the activity of fat-fed hypoactive hamsters (Fig. 2A, open circles), $F(2)$ $=4.476, p<0.05$, but neither group differed from control hamsters. Dietary manipulations did not significantly affect any other aspect of the pattern of running activity.

\section{Neurochemical Determinants of Diet-Induced Changes in} Running Activity

Opiate receptor blockade led to slight nonsignificant decreases in total running activity and did not reliably influence the pattern of spontaneous running (data not shown).

Intraseptal infusion of vehicle led to slight nonsignificant decreases in total running activity compared to preinfusion activity levels and had no distinct effects on the pattern of running (data not shown).

Intraseptal infusion of serotonin neurotoxin facilitated spontaneous running in the fat-fed hamsters (solid circles, Fig. 2). Analysis of covariance with repeated measures revealed significant group differences in distance run, $F(2,16)$ $=3.58, p=0.05$, and pairwise comparisons identified fat-fed hamsters as the source of variance, $t(6)=2.73, p<0.05$ After the toxin, fat-fed hamsters ran as much as the control hamsters. In addition, neurotoxin administration led to significant time effects in the speed of running, number of runs, and duration of pauses ( $p<0.005$ for each, Fig. 2), and the variance in each instance was contributed by the fat-fed group. Thus, intraseptal administration of neurotoxin speeded up the running, increased the number of running bouts, and shortened the pause between the runs.

Three days following the administration of serotonin neurotoxin, there was selective $76.9 \%$ depletion of serotonin from the hippocampus $(p<0.01)$, and a $50.3 \%$ depletion of serotonin from the parietal cortex $(p<0.01)$ but not from the hypothalamus, and there were no changes in regional concentrations of norepinephrine and dopamine (Fig. 3). Eight days following the administration of serotonin neurotoxin, food-restricted hamsters had 1.4 times greater concentration of septal serotonin $(p<0.01$, ) and 1.6 times greater concen- 


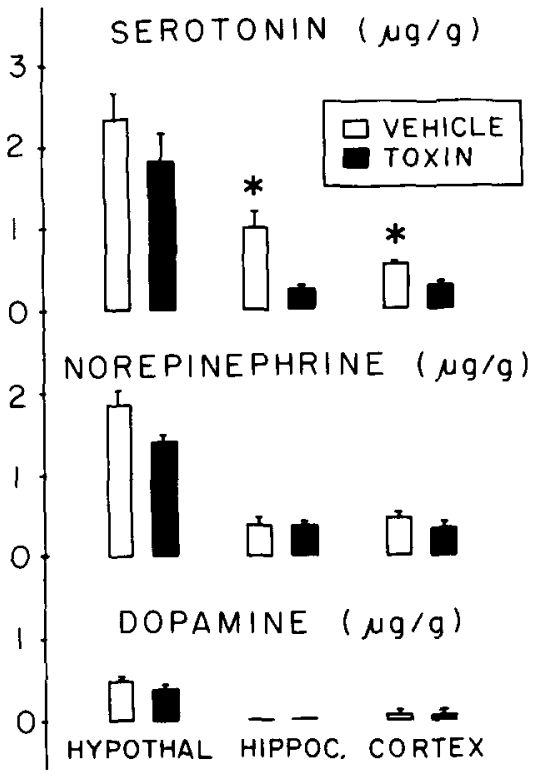

FIG. 3. Concentrations of serotonin, norepinephrine, and dopamine in hypothalamus, septum, hippocampus, and parietal cortex of food-restricted hamsters (solid bars), control-diet hamsters (open bars), and fat-fed hamsters (cross-hatched bars) eighteen days after intraseptal administration of vehicle and eight days after intraseptal administration of serotonin neurotoxin. Brackets denote S.E.M.s.

tration of septal dopamine ( $p<0.05$, Fig. 4) than the other two dietary groups. Neurochemical data from Experiments 1 and 2 showed consistency in pattern, but serotonin and norepinephrine concentrations in the hypothalamus and hippocampus were between 30 and $50 \%$ lower in tissues from Experiment 1 than in tissues from Experiment 2.

\section{DISCUSSION}

Dietary manipulations employed in this study were effective in producing significant displacements of the body energy content of mature female hamsters from the norms displayed by the control group. Supplementation of the standard chow diet with a high-fat diet induced obesity while food restriction led to a $4.5 \%$ drop in body fat content of foodrestricted hamsters. Diet-induced body energy changes were followed by reciprocal changes in the levels of voluntary activity. We have thus been able to induce body energyassociated changes in activity which are frequently reported in cross-sectional studies as associations between the variables of weight $(4-6,37)$, age $(8)$, or body composition (4-7) on one hand and activity levels on the other.

We have been able to clarify the neurochemical mediation of bioenergetic hypoactivity but not of bioenergetic hyperactivity. Our data supported only the hypothesis that serotonergic neurons innervating the hippocampus mediate the hypoactivity induced by obesity. Intraseptal administration of serotonin neurotoxin which has previously been shown to damage serotonin fibers innervating the hippocampus in the rats $(42,43)$, converted the obese hamsters from hypoactive to normally active without an effect on activity levels of control or food-restricted animals. The neurotoxin

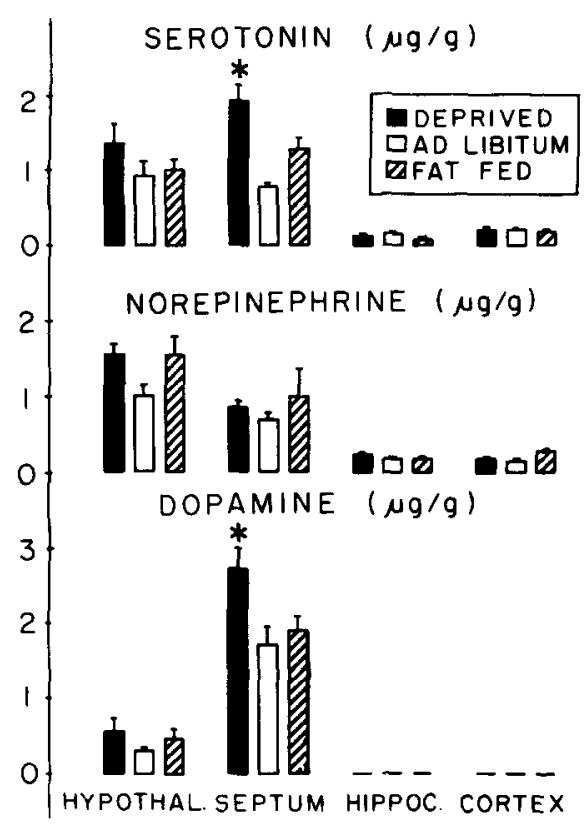

FIG. 4. Concentrations of serotonin, norepinephrine, and dopamine in hypothalamus, hippocampus, and parietal cortex of hamsters which have received three days earlier an intraseptal infusion of either the vehicle (open bars) or serotonin neurotoxin (solid bars). Brackets denote S.E.M.s.

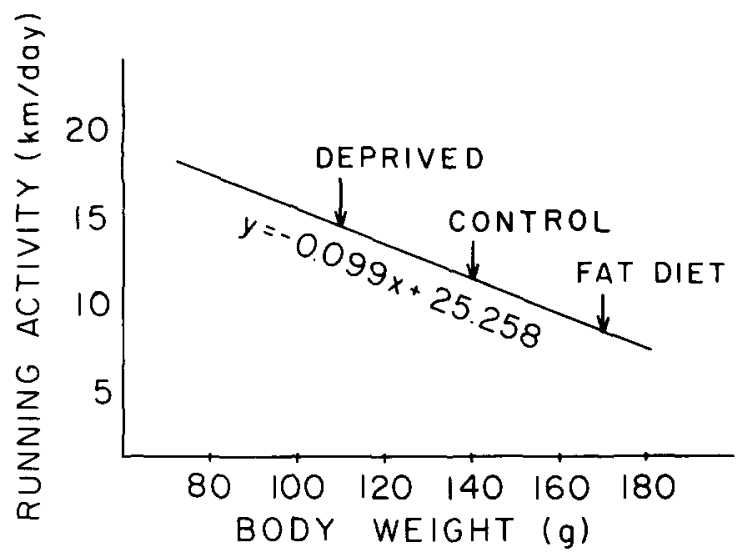

FIG. 5. Changes in daily levels of spontaneous running activity as a function of age-associated increases in body weight in mature female hamsters [redrawn after Borer and Kaplan (8), with permission from Pergamon Press]. Arrows denote activity levels of food-restricted (deprived) hamsters, control hamsters fed chow, and fat-fed hamsters that were recorded in the present study (Fig. 2).

appeared to produce this effect by selective facilitation of running speed and by inducing an increase in the number of running bouts and a decrease in the duration of pauses. These behavioral changes were associated with significant depletion of hippocampal and cortical serotonin which were specific for this neurotransmitter (Figs. 3 and 4) and for the brain regions known to be innervated by serotonergic fibers originating in the nucleus raphe medianus $(20,31)$. 
Our data did not support the second of our two hypotheses, that endogenous opiates are necessary for the expression of hyperactivity in hamsters that are in negative energy balance. Although naloxone administration produced changes in running activity in the expected direction, the magnitude of these changes did not reach statistical significance. We suspect that the age of the animals used in the present study may account for the failure of endogenous opiates to significantly influence running behavior as they were shown to do in 100-day-old hamsters. Activity levels are greatly reduced in aged female hamsters, and are not affected by opiate receptor blockade (33). Since energy deprivation facilitated running in our older hamsters but opiate blockade did not have an effect, the hypothesis that endogenous opiates mediate hyperactivity in hamsters when they are in negative energy balance, is untenable. Other, yet to be identified, neural mediators control such hyperactivity.

We see several significant implications of our observations. First that the reciprocal relationship between the body energy balance and spontaneous physical activity has important nonhomeostatic consequences. Hypoactivity that is induced by accumulation of excess body energy will act to augment and perpetuate obesity once it has been attained. Conversely, a negative energy balance will induce hyperactivity and even further jeopardize the energy balance. Thus, spontaneous running is clearly not a compensatory negative feedback process in the service of energy regulation. It contributes to deviations rather than to corrections of energy balance.

This insight may have implications for humans as well. There is a considerable amount of interest in the possible contributions of spontaneous physical activity to the development and maintenance of human obesity. Early observational studies suggested that obese human infants (36), obese human adolescents $(15,28,39)$, and obese human adults $(2,16)$ engage in less voluntary physical activity than their normal-weight counterparts. These studies favored the interpretation that obese humans, like obese rodents, are hypoactive, and that this hypoactivity may at least contribute to the maintenance, if not to the development, of obesity. Studies demonstrating concurrent development of obesity and hypoactivity in genetically obese rodents, suggested that both changes, obesity and hypoactivity, may reflect simultaneous developmental expression of a common genetic trait $(40)$.

This hypothesis has been challenged by studies using direct measures of energy expenditure as a function of body mass which show that under controlled laboratory circumstances, movement of a heavier body has a higher bioenergetic cost than a movement of lighter body. This evidence was used to argue that the higher bioenergetic cost of movement of a heavier body is not likely to lower the energy balance in obese individuals.

The obvious resolution of the conflicting outcomes would be to perform a bioenergetic study in a naturalistic setting, examining the total spontaneously generated energy expenditure in individuals which display significant deviations from normative energy balance. In a recent attempt at this kind of experiment, results had very high variances but suggested that obese individuals selected low intensity activities more often than the normal weight individuals (1).

It would be of interest to determine whether the association of compulsive recreational running and dieting (44) reflects biological relationships between altered energy balance and levels of spontaneous physical activity (22) rather than psychopathology as these changes are often characterized $(26,30)$. If, indeed, the association of obesity and hypoactivity on one hand, and of negative energy balance and hyperactivity on the other, reflect general biological relationships, then this insight would simplify attempts to correct deviations in human energy balance. Physical activity will need to be linked with external incentives to motivate obese individuals to use this avenue of increased energy expenditure, and negative energy balance will first have to be corrected before the feed-forward contribution of physical activity to energy deficit could be curbed.

The third implication of the biological effects of altered energy balance on spontaneous running activity is its connection with the process of aging. Mature rodents show slow but systematic increases in body weight during the midportion of their life span, and weight losses toward the end of the life span (41). A similar pattern of age-associated changes have been reported in the humans (25). In the hamster, the reciprocal association between the weight gain of maturity and the levels of spontaneous activity is established as early as 7 weeks of life when the animals weigh about 75 to $80 \mathrm{~g}$ (8). A very orderly relationship between weight gain and increasing hypoactivity predicts running levels as a function of weight throughout hamster adult life span [(8), Fig. 5]. This relationship then suggests that weight gains are programmed during the mid-portion of rodent life span and are a natural manifestation of aging

Data obtained in the present study confirm this prediction. Food-restricted animals show the hyperactivity characteristic of younger, lighter hamsters, while hypoactivity of fat-fed hamsters mimics the hypoactivity of older, heavier hamsters (Fig. 5). Viewed from this perspective, excessive weight gain can be interpreted as an acceleration, and weight loss as a reversal, of the aging process for the variables of body mass and spontaneous activity. The relevance of weight-activity relationship to the aging process is affirmed by our demonstration of serotonergic link in this process, and by demonstration by others (38) of the role of serotonin in some other manifestations of aging. Further, by observations of others, that food restriction extends the life span in a number of different species of animals (46).

The present study confirms our hypothesis that septohippocampal interconnections link the information on body energy balance with the spontaneous running behavior. In addition, our study shows that a serotonergic component of this circuit mediates diet-induced hypoactivity. The most probable origin of this inhibitory serotonergic influence over spontaneous running activity is the mesolimbic serotonergic projection to the hippocampus and the cortex from the nucleus raphe medianus $(20,31)$.

An unexpected finding of the present study was that food restriction was associated with significant increases in serotonin and dopamine content within the septum-MPOA region. These changes could possibly be related to compensatory inhibition of resting metabolic rate $(21,29)$ by which underweight hamsters regulate their energy balance (7) or any of other behavioral and physiological consequences of food restriction.

In conclusion, we have shown that experimental alteration of body fat content in mature hamsters leads to changes in spontaneous running activity which can be related to changes in the function of serotonergic neurons innervating the hippocampus. We believe that these data represent the first demonstration of neurochemical basis for hypoactivity which results from obesity in a mature mammal. The signifi- 
cance of these findings is in revealing a neurochemical change in brain function that is a probable mediator of reduction in the amount of spontaneous physical activity induced by obesity. Such dietarily-induced changes in physical activity may contribute to perpetuation of obesity and may represent an acceleration of the natural aging process.

\section{ACKNOWLEDGEMENTS}

This research was supported, in part, by the National Science Foundation grant DCB 85-02902. Gift of naloxone by E. I. Du Pont de Nemours is gratefully acknowledged. Joel Grinker and Terry Robinson provided comments which helped improve the manuscript.

\section{REFERENCES}

1. Blair, D.; Buskirk, E. R. Habitual daily energy expenditure and activity levels on lean and adult-onset and childhood-onset obese women. Am. J. Clin. Nutr. 45:540-550; 1987.

2. Bloom, W. L.; Eidex, M. F. Inactivity as a major factor in obesity. Metabolism 16:679-684; 1967.

3. Blumenthal, J. A.; O'Toole, L. C.; Chang, J. L. Is running an analogue of anorexia nervosa? JAMA 252:520-523; 1984.

4. Borer, K. T. Characteristics of growth-inducing exericse. Physiol. Behav. 24:713-720; 1980.

5. Borer, K. T. The nonhomeostatic motivation to run. In: Morrison, A. R.; Strick, P. L., eds. Changing concepts of the nervous system. New York: Academic Press; 1982:539-567.

6. Borer, K. T. The reciprocal relation between endurance physical activity and body fatness. In: Hansen, B. C., ed. Controversies in obesity. New York: Praeger Scientific; 1983:255273.

7. Borer, K. T.; Allen, E. R.; Smalley, R. E.; Lundell, L.; Stockton, J. Recovery from energy deficit in golden hamsters. Am. J. Physiol. 248:R439-R446; 1985.

8. Borer, K. T.; Kaplan, L. R. Exercise-induced growth in golden hamsters: Effects of age, weight, and activity level. Physiol. Behav. 18:29-34; 1977.

9. Borer, K. T.; Kelch, R. P.; Peugh, J.; Huseman, C. Increased growth hormone and somatic growth in adult hamsters with hippocampal transections. Neuroendocrinology 29:22-33; 1979.

10. Borer, K. T.; Kelch, R. P.; White, M. P.; Dolson, L.; Kuhns, L. $R$. The role of septal area in the neuroendocrine control of growth in the adult golden hamster. Neuroendocrinology 23:133-150; 1977 .

11. Borer, K. T.; Peters, N. L.; Kelch, R. P.; Tsai, A. C.; Holder, $\mathrm{S}$. Contribution of growth, fatness, and activity to weight disturbance after septohypothalamic cuts in adult hamsters. J. Comp. Physiol. Psychol. 93:907-918; 1979.

12. Borer, K. T.; Potter, C. D.; Fileccia, N. Basis for the hypoactivity that accompanies rapid weight gain in hamsters. Physiol. Behav. 30:389-397; 1983.

13. Borer, K. T.; Shapiro, B.; Vinik, A. I. A role for somatostatin in the control of hamster growth. Brain Res. Bull. 11:663-669; 1983.

14. Brownstein, M.; Arimura, A.; Sato, H.; Schally, A. V.; Kizer, J. S. The regional distribution of somatostatin in the rat brain. Endocrinology 96:1456-1461; 1975.

15. Bullen, B. A.; Reed, R. B.; Mayer, J. Physical activity of obese and non-obese adolescent girls appraised by motion picture sampling. Am. J. Clin. Nutr. 14:211-223; 1964.

16. Chirico, A. M.; Stunkard, A. J. Physical activity and human obesity. N. Engl. J. Med. 263:935-940; 1960.

17. Collier, G. H. Work: A weak reinforcer. Trans. NY Acad. Sci. 32:557-576; 1970.

18. Collier, G.; Hirsch, E. Reinforcing properties of spontaneous activity in the rat. J. Comp. Physiol. Psychol. 77:155-160; 1971.

19. Collier, G.; Hirsch, E.; Levitsky, D.; Leshner, A. I. Effort as a dimension of spontaneous activity in rats. J. Comp. Physiol. Psychol. 88:89-96; 1975.

20. Conrad, L. C. A.; Leonard, C. M.; Pfaff, D. W. Connections of the median and dorsal raphe nuclei in the rat. An autoradiographic and degeneration study. J. Comp. Neurol. 156:179-216; 1974.
21. Cooper, K. E.; Cranston, W. L.; Honour, A. J. Effects of intraventricular and intrahypothalamic injection of noradrenaline and 5-HT on body temperature in conscious rabbit. J. Physiol. $181: 852-864 ; 1965$.

22. Crisp, A. H. The possible significance of some behavioral correlates of weight and carbohydrate intake. J. Psychosom. Med. $11: 117-131 ; 1967$

23. Dill, D. B.; Soholt, L. F.; Morris, J. D., Jr. Wheel running of kangaroo rats, Dipodomys merriami, as related to food deprivation and body composition. J. Appl. Physiol. 44:17-20; 1978.

24. Duncan, D. B. Multiple range and multiple F tests. Biometrics $11: 1-42 ; 1955$.

25. Forbes, G. B.; Reina, J. C. Adult lean body mass declines with age: some longitudinal observations. Metabolism 196:653-663 1970.

26. Gull, W. W. Anorexia nervosa (apepsia hysterica, anorexia hysterica). Trans. Clin. Soc. (Lond.) 7:22-28; 1874.

27. Horwitz, W., ed. Meat and meat products, 24.005. Crude fat or ether extract-official final action. In: Official methods of analysis of AOAC. 13th ed. Washington, DC: American Organic Analytic Chemists; 1980:132-370.

28. Johnson, M. L.; Burke, B. S.; Mayer, J. Relative importance of inactivity and overweight in the energy balance of obese high school girls. Am. J. Clin. Nutr. 4:37-44; 1956.

29. Kalivas, P. W.; Nemeroff, C. B.; Miller, J. S.; Prange, A. J., Jr. Microinjection of neurotensin into the ventral tegmental area produces hypothermia. Evaluation of dopaminergic mediation. Brain Res. 326:219-227; 1985.

30. Kron, L.; Katz, J. L.; Gorzynski, G.; Weiner, H. Hyperactivity in anorexia nervosa: A fundamental clinical feature. Comp. Psychiatry $19: 433-440 ; 1978$.

31. Lorens, S. A.; Guldberg, H. C. Regional 5-hydroxytryptamine following selective midbrain raphe lesions in the rat. Brain Res. $78: 45-56 ; 1974$

32. Moskowitz, M. J. Running wheel activity in the white rat as a function of combined food and water deprivation. J. Comp. Physiol. Psychol. 52:621-625; 1959 .

33. Nichols, J. F. The effects of age and acute submaximal exercise on metabolic and hormonal characteristics of hamsters. Ph.D. dissertation, University of Michigan, 1985.

34. Potter, C. D.; Borer, K. T.; Katz, R. J. Opiate-receptor blockade reduces voluntary running but not self-stimulation in hamsters. Pharmacol. Biochem. Behav. 18:217-223; 1983.

35. Rainin Liquid Chromatography catalog; 1985-1986:95.

36. Rose, H. E.; Mayer, J. Activity, caloric intake, fat storage and the energy balance of infants. Pediatrics $41: 18-29 ; 1968$.

37. Sclafani, A.; Rendel, A. Food deprivation-induced activity in dietary obese, dietary lean, and normal-weight rats. Behav. Biol. 24:220-228; 1978 .

38. Simpkins, J. W.; Mueller, G. P.; Huang, H. H.; Meites, J. Evidence for depressed catecholamine and enhanced serotonin metabolism in aging male rats: Possible relation to gonadotropin secretion. Endocrinology 100:1672-1678; 1977.

39. Stefanik, P. A.; Heald, F. P., Jr.; Mayer, J. Caloric intake in relation to energy output of nonobese and obese adolescent boys. Am. J. Clin. Nutr. 7:55-62; 1959.

40. Stern, J. S.; Johnson, P. R. Spontaneous activity and adipose cellularity in the genetically obese Zucker rat (fafa). Metabolism 26:371-380; 1977 
41. Thomas, R. G.; London, J. E.; Drake, G. A.; Jackson, D. E. Wilson, J. S.; Smith, D. M. The golden hamster: Quantitative anatomy with age. Los Alamos: Los Alamos Sci. Lab.; 1979.

42. Waldbillig, R. J.; Weider, G. E.; Clemmons, R. M.; Bartness, T. J. Rostral hypothalamic microinfusions of 5,7-dihydroxytryptamine produce anatomically and neurochemically selective depletions of hippocampal serotonin and increase the influence of estrogen and food deprivation on locomotor activity. Brain Res. 347:149-153; 1985.
43. Williams, J. H.; Azmitia, E. C. Hippocampal serotonin reuptake and nocturnal locomotor activity after microinjection of 5,7-DHT in the fornix-fimbria. Brain Res. 207:95-107: 1981.

44. Yates, A.; Leehey, K.; Shisslak, C. M. Running-an analogue of anorexia? N. Engl. J. Med. 303:251-255; 1983.

45. Yen, T. T. T.; Acton, J. M. Locomotor activity of various types of genetically obese mice. Proc. Soc. Exp. Biol. Med. 140:647$650 ; 1972$.

46. Young, V. R. Diet as modulator of aging and longevity. Fed. Proc. 38:1994-2000; 1979. 